\title{
Prediction of the failure locus of CPEEK composites under transverse compression and longitudinal shear through computational micromechanics
}

\author{
Essam Totry , Carlos Gonzalez , Javier LLorca \\ Departamento de Ciencia de Materiales, Universidad Politecnica de Madrid, E. T. S. de Ingeniems de Caminos, 28040 Madrid, Spain \\ Instituto Madrileno de Estudios Avanzados en Materiales (IMDEA-Materiales), E. T. S. de Ingeniems de Caminos, 28040 Madrid, Spain
}

\begin{abstract}
A B S T R A C T
The potential of computational micromechanics to predict the failure locus of a unidirectional C/PEEK composite subjected to transverse compression and longitudinal shear was established. Numerical simulations were compared with the experimental results of Vogler and Kyriakides [Vogler TJ, Kyriakides S. Inelastic behavior of an AS4/PEEK composite under combined transverse compression and shear. Part I: Experiments. Int J Plasticity 1999;15:783-806], which contain detailed information of the matrix and fiber properties as well as the failure micromechanisms during multiaxial loading. Analyses were based in the finite element analysis of a three-dimensional representative volume element of the lamina microstructure and included the main deformation and failure mechanisms observed experimentally, namely matrix shear yielding and interface decohesion. In addition, the numerical predictions of the failure locus for composites with strong and weak interfaces were compared with those obtained by current phenomenological failure models to establish the accuracy and range of validity of these criteria.
\end{abstract}

Keywords:

A. Polymer-matrix composites

B. Mechanical properties

B. Modeling

C. Failure criterion mainly aimed to study the effect of microstructural factors (fiber orientation, interface strength, etc.) on the onset and progression of damage upon loading. Another interesting application in this field the prediction of the failure locus of PMC lamina subjected to multiaxial stress states, an important and controverted problem. A recent effort to assess the state-of-the-art developments in this matter, the World Wide Failure Exercise , compared the results provided by 19 different failure criteria with benchmark experimental results on carbon- and glass-fiber laminates. The comparison showed that the predictions of many theories differed significantly from the experimental results (particularly under multiaxial stress states involving fracture by matrix and/or fiber compression), and ranked the available theories according to their ability to predict (a) biaxial lamina strength, (b) initial and final biaxial laminate strength, and (c) deformation of laminates. Precise conclusions regarding to the theory which best reproduces the physical failure mechanisms as well as the mechanical strength were limited by the scarcity of experimental results for multiaxial stress states, the experimental scatter (which sometimes impedes discrimination among the different theoretical predictions), and the phenomenological nature of most models.

Computational micromechanics stands as a very useful tool in this area as it can provide "virtual tests" which can be used to check the different failure criteria available in the literature. In addition, results of virtual tests are cheaper and free from experimental constrains (buckling, free edge effects, failures at end constraints). However, this methodology should be validated previously against experimental results of PMC lamina subjected to multiaxial stress states and this is the main objective of this 
paper. To this end, the failure locus of a unidirectional C/ PEEK composite subjected to transverse compression and longitudinal shear was computed and compared with the experimental results of Vogler and Kyriakides [1], which contain detailed information of the matrix and fiber properties as well as the failure micromechanisms during multiaxial loading. The predicted failure locus was compared with the predictions of Puck and LaRC failure criteria and the influence of matrix and interface properties on the accuracy of these criteria was discussed.

\section{Summary of experimental results}

Vogler and Kyriakides carried out a detailed experimental study of the mechanical behavior of a composite lamina made up of a PEEK matrix unidirectionally reinforced with $60 \mathrm{vol} \%$ AS4 carbon fibers. In addition to tests under transverse compression and longitudinal shear, biaxial tests under three different loading paths (shear followed by compression, compression followed by shear and proportional shear-compression loading) were performed and the failure strength was reported in all cases. While all the specimens fractured along planes parallel to the fibers, the orientation depended on the loading mode. Specimens tested in pure shear failed by splitting near the interface with the end plates and hackles (indicative of shear failure) were found on the fracture surfaces. Specimens tested under pure compression tended to fail within the test section along planes oriented approximately at $45^{\circ}$ with the loading axis although the precise inclination was not measured. Specimens subjected to biaxial loading paths failed in one of these two modes. The former was dominant under small compressive stresses and it was found that the shear strength increased slightly with the compressive stress. The remaining specimens failed in a similar mode to the specimens tested under transverse compression and hackles were also observed on the matrix fracture surfaces.

The experimental values of the compressive $\left(\mathrm{er}_{2}\right)$ an shear $\left(\mathrm{T}_{12}\right)$ stresses at failure are plotted in Fig. 6, and they were independent of the loading path, in agreement with the predictions of numerical simulations . The experimental scatter was limited and thus this set of experimental data is ideal to check the accuracy of the predictions obtained by computational micromechanics. In addition, the authors reported the matrix and fiber mechanical properties, which are necessary for the numerical simulations presented below. It was finally noted by the authors that the failure stresses in Fig. 6 should be interpreted as lower bounds of the actual strength (particularly under shear-dominated fracture) because of the end plate effects. The differences should be, however, small in view of the low tangent moduli of the responses at large strains.

It should be noted that Hsu carried out numerical simulations of these experiments, which were aimed at explaining the lack of influence of the loading path on the yield stress of the composite. They could not provide an estimation of the composite strength because the RVE was very simple (fibers were assumed to be distributed in a regular hexagonal pattern within the matrix) and damage by interface failure was not included. These limitations were overcome in the numerical model presented below.

\section{Numerical model}

\subsection{Representative volume element of the microstructure}

The RVE of the lamina microstructure is a prism, with a square section of dimensions $L x$ Lin the 23 plane perpendicular to the fibers (Fig. 1). The fiber centers in this plane were generated randomly and sequentially following the strategy of the random sequential adsortion algorithm,

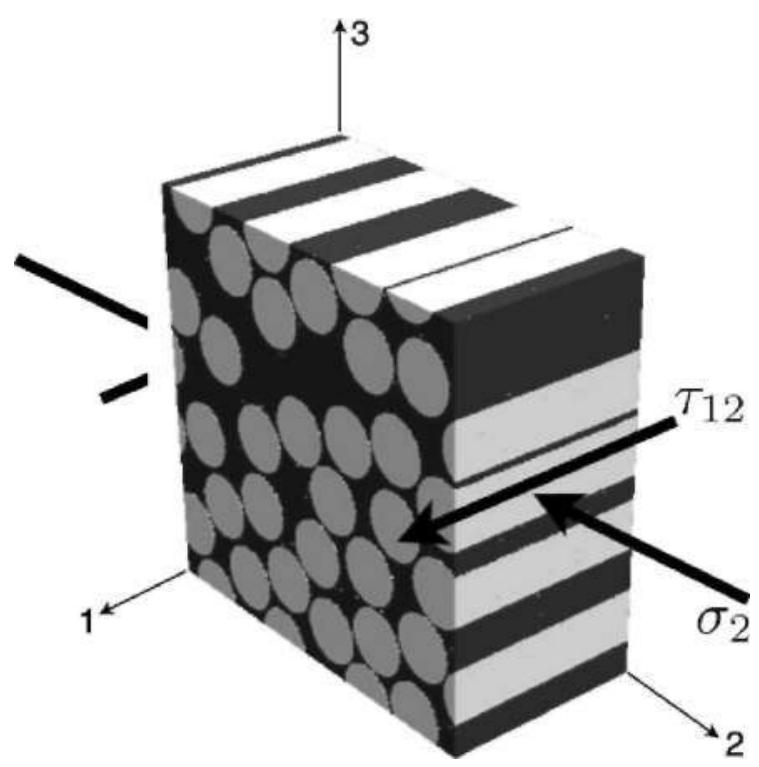

Fig. 1. Schematic of the representative volume element of the lamina microstructure subjected to transverse compression $\left(\mathrm{Cx}_{2}\right)$ and longitudinal shear $\left(\mathrm{TI}_{2}\right)-$

of 10 urn in diameter were included within the RVE with a volume fraction of $60 \%$. It was assumed that the lamina microstructure was given by an indefinite translation of this RVE along the 2 and 3 axes to eliminate surface effects and, thus, the fiber positions within the RVE kept this periodicity condition. To this end, fibers intersecting the RVE edges were split into an appropriate number of parts and copied to the opposite sides of the square RVE to create the periodic microstructure. Finally, the RVE was extruded along the fiber axis to obtain the three-dimensional prism. The RVE was very thin along the 1 axis (t) because no deformation gradients occur along this axis during transverse compression and longitudinal shear. ${ }^{1}$ Previous simulations of PMC lamina subjected to transverse compression and transverse shear have demonstrated that this size of the RVE is large enough to capture accurately the maximum stress supported by the composite lamina, particularly if the results obtained with various fiber realizations are averaged. It should be noted, however, that damage localized within a band in the RVE after the maximum in stress and that much larger RVEs would be necessary to capture accurately the behavior in the postpeak regime.

\subsection{Finite element model}

The prismatic RVE was discretized for the analysis with the finite element method. The model volume (matrix and fibers) was meshed using modified quadratic 10-node tetrahedra (C3D10M in Abaqus ) using an adaptive automatic meshing algorithm. The modified elements contain three extra internal degrees of freedom (corresponding to an internal node) with respect to the standard tetrahedra. They were originally developed to alleviate some problems of the standard 10-node tetrahedra for contact problems (particularly in uniform pressure situations), but it has been shown that the extra internal node increases the accuracy to reproduce the strain gradient in the matrix between closely packed fibers locking.

In addition, they exhibit minimal shear and volumetric

\footnotetext{
1 In fact, it was checked that the results of the numerical simulations were independent of the thickness RVE, whose length was only limited by the aspect ratio
} of the finite elements. 
In order to take into account the effect of interface fracture on the composite response, interface elements were included between matrix and fibers throughout the model. The interface element is made up of two quadratic (6-node) triangular surfaces that connect the faces of two adjacent quadratic tetrahedra. The two triangular surfaces of the interface element lie together in the initial configuration (zero thickness) and separate as the adjacent solid elements deform. The relative displacement of the element faces generate normal and shear stresses depending on the constitutive equation of the cohesive crack, which is independent of the element formulation. The interface elements are compatible with C3D10M solid elements in Abaqus and were programmed as a UEL subroutine.

Periodic boundary conditions were applied to the RVE surfaces to ensure continuity between neighboring RVEs (which deform like jigsaw puzzles). The periodic boundary conditions can be expressed in terms of the displacement vectors 0$], 0_{2}$ and $0_{3}$ which relate the displacements between opposite faces according to

$\vec{u}\left(0, x_{2}, x_{3}\right)-\dot{u}\left(t, x_{2}, x_{3}\right)-\vec{U}_{1}$,

$\vec{u}\left(x_{1}, 0, x_{3}\right)-\vec{u}\left(x_{1}, L, x_{3}\right)-\vec{U}_{2}$,

$\vec{u}\left(x_{1}, x_{2}, 0\right)-\vec{u}\left(x_{1}, x_{2}, L\right)-\vec{U}_{3}$.

Combined uniaxial compression along the $x_{2}$ axis and longitudinal shear along the fibers $\left\{a_{2}\right.$ and $\mathrm{T}_{12}$, Fig. 1) are imposed with $0_{2}=\left(S_{s},-S_{c}, 0\right)$, where $c 5_{\mathrm{c}}$ and $S_{s}$ stand for the imposed compressive and shear displacements, respectively. The components of 0] and $\mathrm{O}_{3}$ are chosen so that the all the normal and shear forces acting on the RVE surfaces are zero (besides those corresponding to transverse compression and longitudinal shear). The compressive and shear strains are given by $\mathrm{e}_{2}=\ln \{1-S J L\}$ and $\mathrm{y}_{12}=\arctan \left\{\mathrm{c} 5_{\mathrm{s}}\right\}$ $(\mathrm{I}-\mathrm{c5}$ ) $)$, respectively. The corresponding normal and shear stresses were computed from the resultant normal and tangential forces acting on the RVE faces divided by the actual cross-section. Simulations were carried out with Abaqus/Standard within the framework of the small deformation/displacement theory because the emphasis was in the composite strength, which was normally attained at strains below 7\%. Moreover, it was checked in selected cases that simulations carried out within the framework of finite deformations theory provided equivalent results. The average finite element model comprised approximately 25,000 elements and 55,000 nodes.

\subsection{Constitutive equations of matrix, fibers and interface}

Carbon fibers were modeled as linear, thermo-elastic transversally isotropic solids and their elastic constants are given in Table 1. The epoxy matrix was assumed to behave as an isotropic, elasto-plastic solid and the elastic constants are given in Table 2. Plastic deformation was governed by the Mohr-Coulomb criterion and the total matrix strain was given by the addition of the elastic and plastic strain components. The Mohr-Coulomb criterion assumes that yielding takes place when the shear stress acting on a specific plane, $x$, reaches a critical value, which depends on the normal stress $a_{n}$ acting on that plane and takes into account the influence of the triaxiality on the shear yielding of polymers. The corresponding yield surface, written in terms of the maximum and minimum principal stresses $\left\{a_{x}\right.$ and $a_{m}$ ), is given by

Table 1

Fiber elastic constants (from [21])

\begin{tabular}{lllll}
\hline $\mathrm{E},(\mathrm{GPa})$ & $£_{2}=£_{3}(\mathrm{GPa})$ & $\mathrm{v}_{12}=\mathrm{v}_{13}$ & $\mathrm{v}_{2} 3$ & $\mathrm{G}_{12}=\mathrm{G}_{13}(\mathrm{GPa})$ \\
\hline 214 & 26 & 0.280 & 0.445 & 112
\end{tabular}

Table 2

Matrix elastic constants (from [21])

\begin{tabular}{ll}
\hline$E(\mathrm{GPa})$ & $v$ \\
\hline 4.10 & 0.356 \\
\hline
\end{tabular}

$F\left(\sigma_{\mathrm{I}}, \sigma_{\mathrm{III}}\right)=\left(\sigma_{\mathrm{I}}-\sigma_{\mathrm{III}}\right)+\left(\sigma_{\mathrm{I}}+\sigma_{\mathrm{III}}\right) \sin \phi-2 c \cos \phi-0$

$\mathrm{F}(\mathrm{CI}, \mathrm{CIII})=(\mathrm{ci}-<7 \mathrm{~m})+(<7 \mathrm{i}-\mathrm{i}-<7 \mathrm{iii}) \sin ^{\wedge}-2 \cos { }^{\wedge}=0$, where $\mathrm{c}$ is the cohesion and $<p$ the friction angle. They were assumed to be constant and independent of the accumulated plastic strain. A non-associative flow rule was used to compute the directions of plastic flow in the stress space and the corresponding potential flow $\mathrm{G}$ was expressed as

$$
\begin{aligned}
& \mathrm{G} \text { was expressed as } 4\left(1-e^{2}\right) \cos ^{2} \Theta-(2 e-1)^{2} \\
& \begin{aligned}
\left.2\left(1-e^{2}\right) \cos \Theta+(2 e-1) \sqrt{4}\left(1-e^{2}\right) \cos ^{2} \Theta-5 e^{2}-4 e\right) \\
\quad \times \frac{3-\sin \phi}{6 \cos \phi},
\end{aligned}
\end{aligned}
$$

in which $\mathrm{e}=(3-\sin \$) /(3+\sin \langle>)$ and 0 is obtained from

$0=1 \arccos V j X$,

where $J_{2}$ and $/ 3$ are, respectively, the second and the third invariants of the deviatoric stress tensor. More details about the numerical implementation of the Mohr-Coulomb model

Quinson measured the yield point of various glassy polymers subjected to different triaxiality levels (uniaxial tension and compression, simple shear and plane strain compression) and reported friction angles in the range $7^{\circ}-15^{\circ}$. They are in agreement with the magnitude of $15^{\circ}$ obtained from the deviation of the fracture plane from the maximum shear stress plane in FRP subjected to transverse compression and thus $\langle j\rangle=15^{\circ}$ was chosen for the PEEK matrix in this investigation. The friction angle $\langle j\rangle$ controls the hydrostatic pressure sensitivity of the polymeric matrix. The Tresca yield criterion (which is independent of the hydrostatic stress component) is recovered when $\langle j\rangle=0$ while the yield stress of the polymeric matrix under compression and the trend to form shear bands increases with $\langle j\rangle$. Regarding to the yield stress under pure shear, Vogler and Kyriakides carried out tests of the neat PEEK matrix but indicated that the inelastic matrix properties within the composite were different , and this has been corroborated recently through nanoindentation . The matrix cohesion $c$ controls the composite strength under pure shear and, therefore, in absence of accurate and reliable data, $c$ was chosen equal to $79.5 \mathrm{MPa}$, the strength of the composite in shear. This hypothesis takes into account that failure under longitudinal shear occurs through the matrix in agreement with the experimental observations

Fiber/matrix interfaces were simulated through a cohesive crack model. In the absence of damage, the interface behavior was linear with an initial stiffness $K_{t}\left(10^{5} \mathrm{GPa}\right)$, which is a numerical parameter large enough to ensure the displacement continuity at the interface and to avoid any modification of the stress fields around the fibers in the absence of damage. The linear behavior ends at the onset of damage, which occurs when the traction acting on the interface reaches the interface strength, $t_{c}$. This is expressed mathematically as

$\sqrt{\left\langle t_{n}\right\rangle^{2}+t_{t 1}^{2}+t_{i 2}^{2}}-t_{\mathrm{c}}$.

where $\mathrm{t}_{\mathrm{n}}, t_{n}$ and $t_{t 2}$ are, respectively, the normal and tangential stresses transferred by the interface. () stand for the Macaulay brackets, which return the argument if positive and zero otherwise, because compressive normal stresses do not open the crack. 


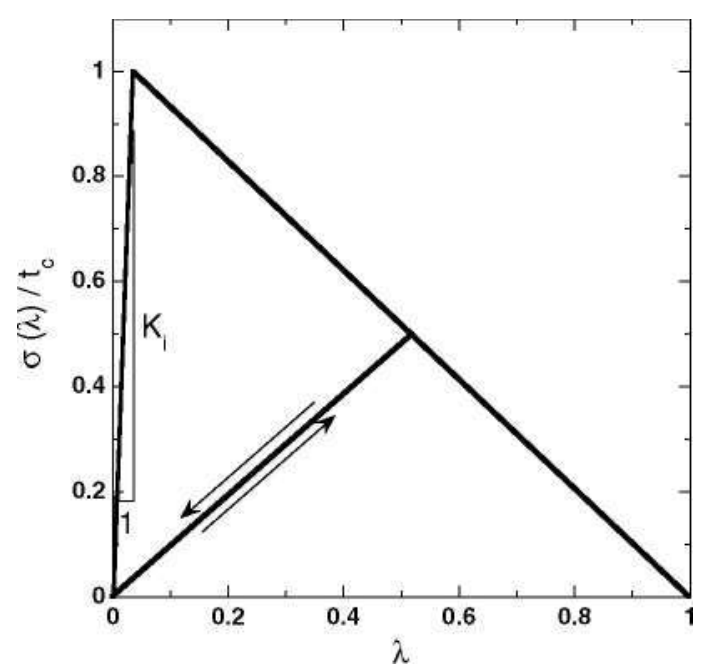

Fig. 2. Function $o(X)$. Notice the stiffness degradation upon unloading once the maximum interfacial strength has been reached.

Once damage began, the stresses transferred through the interface were expressed by

$t_{n}-\frac{\hat{c} \Phi}{\hat{c} \Delta u_{\mathrm{n}}}, \quad t_{n 1}=\frac{\hat{c} \Phi}{\hat{c} \Delta u_{t 1}}, \quad t_{i 2}=\frac{\hat{c} \Phi}{\hat{c} \Delta u_{t 2}}$,

where $<P$ is an elastic potential given by

$\Phi\left(\Delta u_{\mathrm{n}}, \Delta u_{t 1}, \Delta u_{t 2}\right)-\Delta u_{c} \int_{0}^{\lambda} \sigma\left(\lambda^{\prime}\right) \mathrm{d} \imath^{\prime}$

and $A u_{n}, A u_{n}$ and $A u_{t 2}$ stand for the normal and tangential relative displacements between the crack faces. $\mathrm{Au}_{\mathrm{c}}$ is the critical normal (or tangential) displacement between the crack faces at which all interaction vanishes and $X$ is the generalized crack opening displacement expressed as

$\lambda-\sqrt{\left(\frac{\Delta u_{\mathrm{n}}}{\Delta u_{c}}\right)^{2}+\left(\frac{\Delta u_{t 1}}{\Delta u_{c}}\right)^{2}+\left(\frac{\Delta u_{i 2}}{\Delta u_{c}}\right)^{2} .}$

The function o"(l) (which stands for the normal stress transferred through the crack in the absence of tangential displacements) is plotted in Fig. 2. It includes the progressive degradation of the interface stiffness and strength once the maximum interfacial strength is reached, as indicated in the unloading and reloading paths. More details about the numerical implementation of the model can be found in previous publications

The interface behavior is given by the interface strength, $t_{c}$, and the fracture energy, $\mathrm{r}$, which is the area enclosed under the $a\{l)$ function (Fig. 2), and it is given by

$\mathrm{r},=\mathrm{it}_{\mathrm{c}} \mathrm{Au} \mathrm{u}_{\mathrm{c}}$

The mechanical properties of the interface can be measured from push-out tests performed on thin slices machined from the composite panel. No information was, however, available for the interface properties and thus $t_{c}=112 \mathrm{MPa}$ was chosen so the numerical simulations under transverse compression matched the experimental results. This value is representative of a good fiber/matrix bonding, as indicated by the fracture surfaces of the composite samples. Previous simulations have shown that the interface fracture energy does not influence significantly the composite strength in materials with high good interface strength and $\mathrm{r},=100 \mathrm{~J} / \mathrm{m}^{2}$ was used, a reasonable value for C/epoxy interfaces according to available experimental data

\section{Results}

Six different fiber realizations were generated to build the corresponding RVEs and meshed following the methodology presented in the previous section. The mechanical response was computed under transverse compression, longitudinal shear and biaxial compression and shear using a pseudo-radial loading path in which $55_{5} 5_{C}=-6$. The corresponding stress-strain curves for pure compression and shear are plotted in Fig. 3(a), while those for biaxial compression and shear are plotted in Fig. 3(b). The strength of the composite lamina in compression was slightly higher than that of the unreinforced matrix, $\mathrm{y}^{\mathrm{TM}}$, which was computed in accordance with the Mohr-Coulomb model. Different fiber realizations led variations in the composite strength under uniaxial compression but the maximum difference was below $7 \%$ of the compressive strength. Thus, the average value from the six realizations could be considered as a very good approximation of the compressive strength. The specimens deformed in uniaxial com-
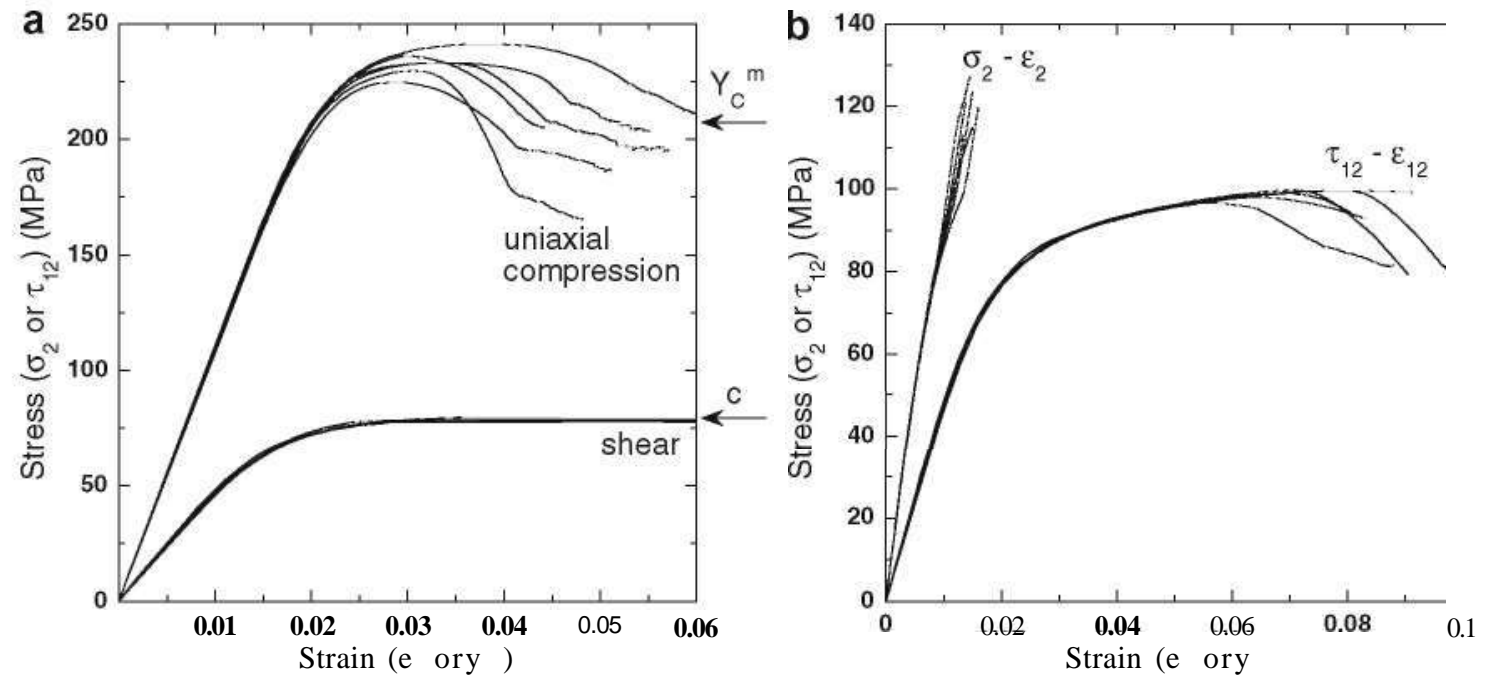

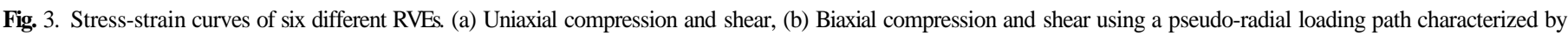
$\& S . I \& C=-6$. The absolute value of the compressive stresses and strains are used in the plots. 
Plastic strain

$0.50 .40 .30 .20 .1 \quad 0$
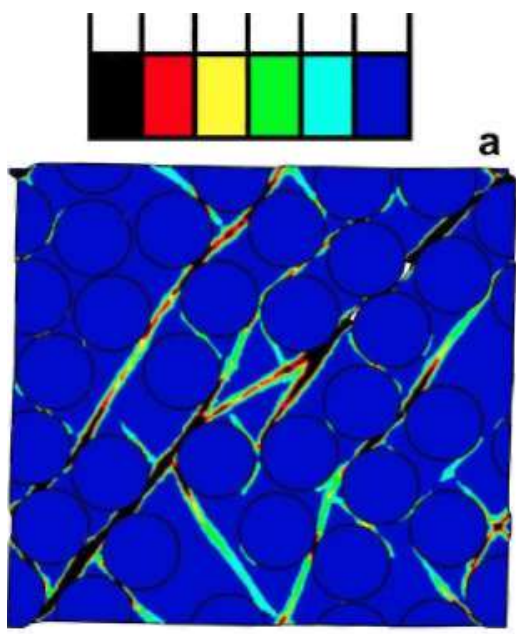

c

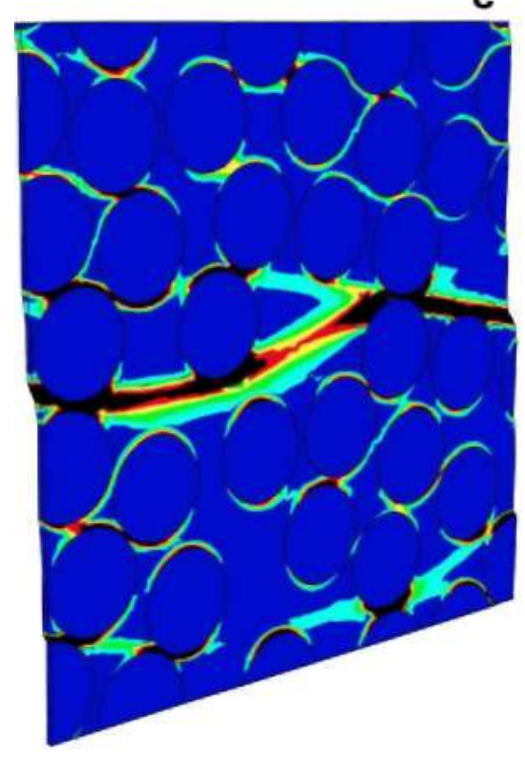

b

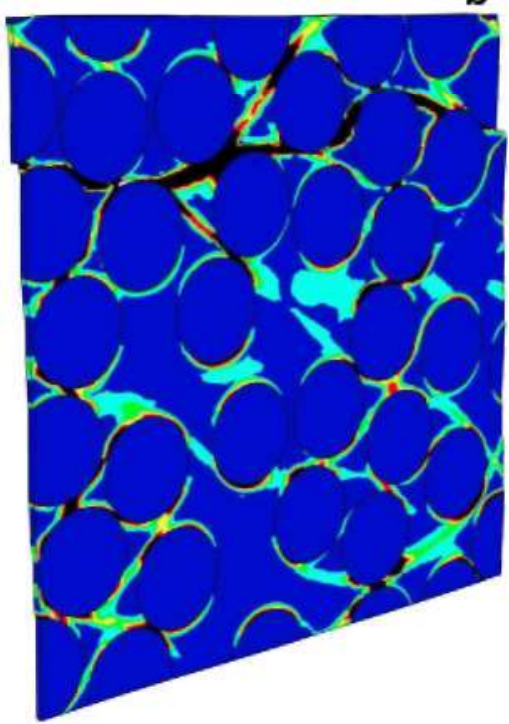

d

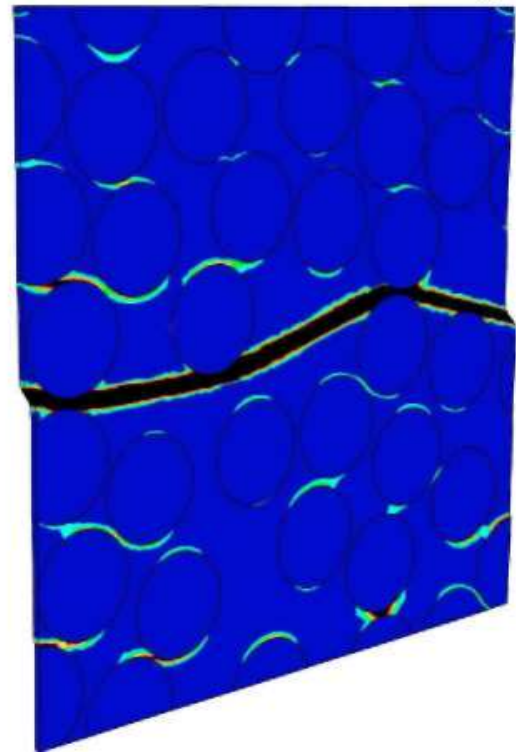

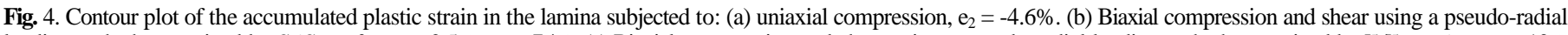

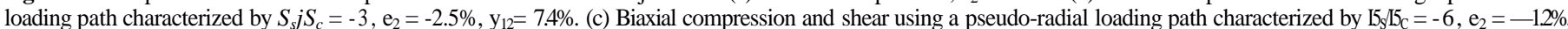

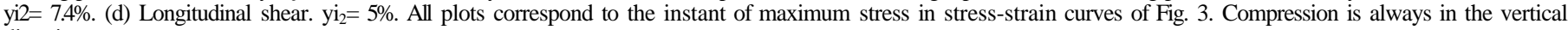
direction.

pression failed by the development of a plastic shear band in the matrix, which was approximately oriented at $50^{\circ}-55^{\circ}$ with the pependicular to the loading axis, in agreement with the experimental observations ( ; Fig. 4(a)). Decohesions at the fiber/ matrix interface were observed in several fibers around the shear band but they appeared after the shear band was formed and thus were a consequence of the strain localization rather than the origin. Obviously, the scatter in the stress-strain curves among different RVEs increased in the post-peak regime because the behavior was controlled by the path and orientation of the shear band in the matrix.

On the contrary, the stress-strain curves of the six realizations under longitudinal shear were practically superposed and presented a wide plateau, which showed that the composite deformed at a constant shear stress equal to the matrix flow stress in shear, $c$. The corresponding deformation pattern can be found in the contour plot of the accumulated plastic strain in the matrix in Fig. 4(d). Deformation was initially localized in a matrix band parallel to the applied shear stress $\mathrm{T}_{12}$ and further loading only increased the shear strain in the band. Comparison among the different RVEs showed that the localization occurred along the shortest matrix path parallel to applied shear stress. Once the band percolated through the RVE, deformation continued at a constant shear stress equal to the matrix cohesion. This deformation micromechanism is in perfect agreement with the experimental observations, which reported that the specimens loaded in longitudinal shear failed by splitting along the fibers. Our constitutive equation for the matrix did not take into account the matrix failure in shear and thus it was not possible to reproduce the final fracture of the lamina under these conditions, though this was not necessary as the focus of the simulations was in the maximum strength.

The stress-strain curves for six different RVEs subjected to compression and shear using a pseudo-radial loading path characterized by $S_{s} / S_{c}=-6$ also showed very little scatter (Fig. 3(b)). 
Failure occurred by the development of a shear band in the matrix parallel to the applied shear stress (Fig. 4(c)), as in the specimens subjected to uniaxial shear. Nevertheless, the compressive stresses led to a more diffuse damage pattern in the matrix and increased the matrix resistance to shear yielding. As a result, the $T_{12}-y-12$ curves showed strain hardening and presented a well-defined maximum in strength which was followed by the localization of the deformation in continuous band throughout the RVE.

The behavior of the lamina subjected to different biaxial loading paths characterized by $5_{3} \Sigma_{C}$ is plotted in Fig. 5 in the $a_{2}-t 2$ stress space. Normal and tangential stresses increased proportionally during elastic deformation upon pseudo-radial deformation and this proportionally disappeared with the onset of plastic deformation in the matrix. Two different failure modes were observed. If $\langle 5$ $5 \mathrm{sg}-4$, the composite failed in shear-dominated mode and deformation was localized in matrix band parallel to the shear stress. It should be noted that the maximum shear strength increased with the compressive stress applied to the lamina while this failure mode was dominant, in agreement with the postulates of the Mohr-Coulomb model. The compressive stresses became dominant as $\left\langle 5_{s}\left(5_{c}\right\rangle-2\right.$ and fracture occurred through a matrix plane whose inclination with respect to the line perpendicular to the compressive stress was in the range $50^{\circ}-55^{\circ}$, depending on the details of the spatial arrangement of the fibers. The transition between both mechanisms was found at $5 k_{5}$ ss -3 , in which the orientation of the fracture surface was in between the behavior dominated by shear or compression, as shown in Fig. 4(b).

The predictions of the failure locus under transverse compression and longitudinal shear obtained by computational micromechanics are plotted in Fig. 6 together with the experimental results of Vogler and Kyriakides . The agreement is excellent for the whole shape of the failure locus and it is worth noting the experimental points in Fig. 6 should be interpreted as lower bounds of the actual strength under shear-dominated fracture because of the end plate effects . It should be noted that the differences between the numerical results and the experiments in the region of shear-dominated failure may also appear because the friction angle chosen for the matrix in the simulations $\left(t p^{=} 15^{\circ}\right)$ was slightly higher than the actual one. The numerical simulations explained the transition from shear failure by splitting along the fibers to compressive failure along an inclined surface parallel to the fibers. The former is characterized by a linear increase of the shear

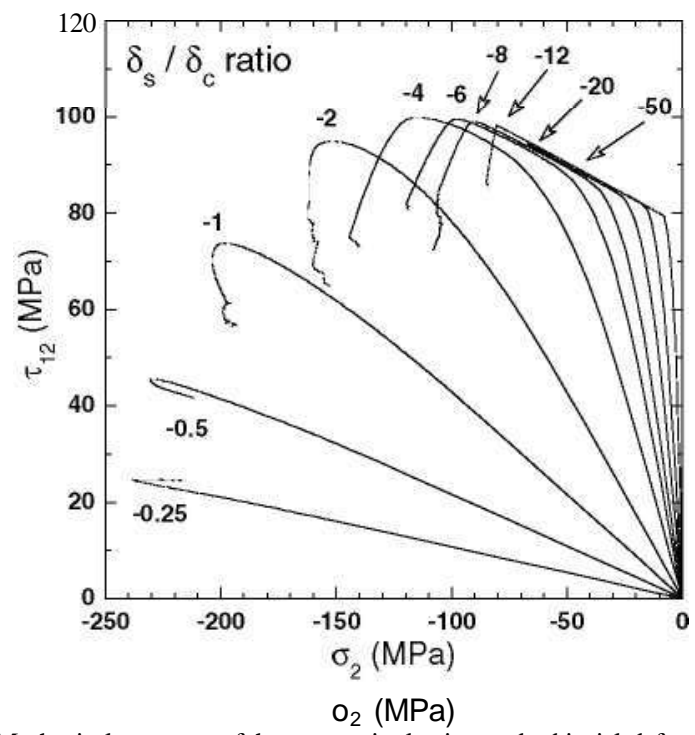

Fig. 5. Mechanical response of the composite lamina under biaxial deformation in the $<\mathrm{T}_{2}-\mathrm{T} 12$ stress space. The corresponding $S J S_{S}$ ratio for each curve is given in the figure.

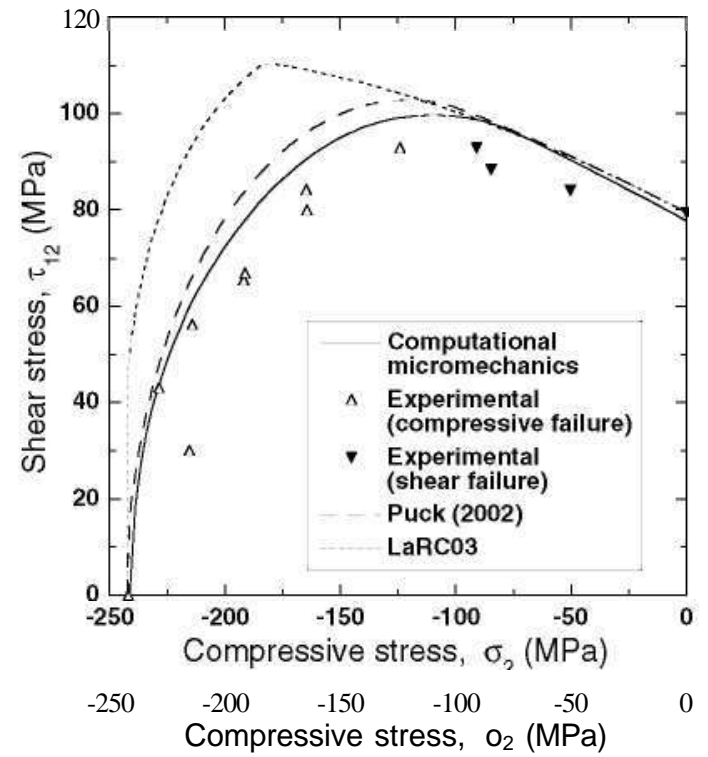

Fig. 6. Failure locus under transverse compression and longitudinal shear. Experimental results [1] and numerical simulations using computational micromechanics as well as predictions of the Puck and LaRCO3 models

strength with the applied compressive stress and the slope depends of the friction angle $\langle j\rangle$. The end of this regime is accompanied by a change in the failure mode to compressive failure, leading to a gentle reduction in the maximum shear strength at failure.

The corresponding failure envelopes corresponding to the Puck and LaRC03 models are also plotted in Fig. 6 for comparison. Both models assume that the composite lamina is isotropic in the 23 plane and that failure under transverse compression and longitudinal shear occurs through surfaces parallel to the fibers. Fracture is caused by the normal and tangential stresses acting on the fracture plane following the Mohr-Coulomb criterion (matrix-dominated failure). The failure surface is determined by computing the critical orientation of the fracture plane for each combination of normal and tangential stresses. The two models differ slightly in their expression of the Mohr-Coulomb failure criterion. Puck proposed

$$
\left(\begin{array}{c}
\tau_{\mathrm{t}} \\
S_{\mathrm{T}}-\eta_{\mathrm{t}} \sigma_{\mathrm{n}}
\end{array}\right)^{2}+\left(\begin{array}{c}
\tau_{\mathrm{I}} \\
S_{\mathrm{L}}-\eta_{\mathrm{l}} \sigma_{\mathrm{n}}
\end{array}\right)^{2}-1,
$$

where $\mathrm{x}_{\mathrm{t}}$ and $\% \backslash$ are, respectively, the transverse and longitudinal shear stresses and $a_{n}$ is the normal stress acting on the fracture plane (Fig. 7). $S_{\mathrm{L}}$ and $\mathrm{S}_{\mathrm{T}}$ stand for the lamina strength under longitudinal and transverse shear. $S_{\mathrm{L}}$ can be measured directly in the laboratory but mechanical tests under transverse (out-of-plane) shear are very difficult to carry out and $\mathrm{S}_{\mathrm{T}}$ is normally from the transverse compressive strength of the lamina $Y_{c}$ by assuming the failure is controlled by the matrix and follows the Mohr-Coulomb model. Under these hypotheses,

$S_{\mathrm{T}}=\frac{Y_{\mathrm{C}}}{2 \tan \theta_{0}}$,

where $0_{o}$ indicates the orientation of the fracture plane under transverse compression with respect to the perpendicular to the loading axis, which is close to $53^{\circ}$ for FRP if matrix failure is dominant. The other parameters in Eq. (10) can be obtained as [19]

$\eta_{\mathrm{t}}=-\frac{1}{\tan 2 \theta_{0}}$ and $\eta_{\mathrm{l}}=\eta_{\mathrm{t}} \frac{S_{\mathrm{l}}}{S_{\mathrm{T}}}$.

The failure locus can be determined by computing the critical orientation of the fracture plane for each combination of normal and tan- 


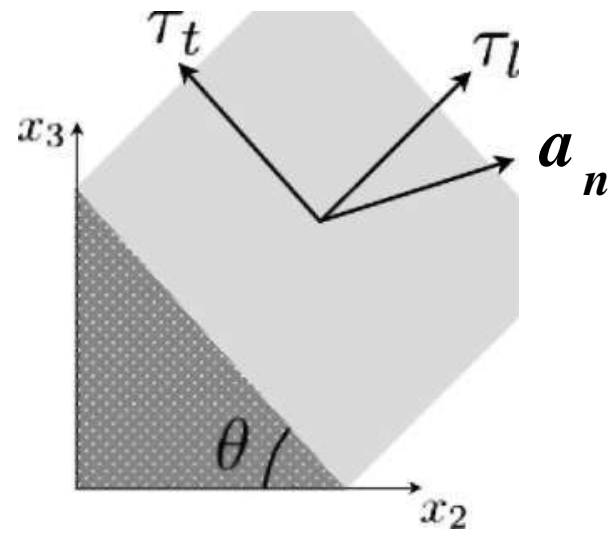

Fig. 7. Schematic of normal and shear stresses acting on the fracture plane according to Puck and LaRC models.

gential stresses once the lamina properties under longitudinal shear and transverse compression are given

Davila followed a similar strategy to obtain the failure locus under transverse compression and longitudinal shear starting from another approximation based on the Mohr-Coulomb model$$
\text { ST }
$$

$$
=1 \text {, }
$$

which assumes that the compressive stresses acting on the fracture plane reduce the effective shear stresses rather than increase the shear strength.

Both models (Puck and LaRC03) reproduce adequately the two main failure mechanisms (compressive and shear-dominated failure) which were found in the experiments and in the numerical simulations. Nevertheless, the Puck model (which also stands for the $\mathrm{LaRCO} 4$ criteria) is able to predict accurately the failure surface in the whole range of biaxial stresses. On the contrary, LaRC03 criteria does not capture adequately the transition between both failure mechanisms and tends to overestimate the composite strength.

\section{Failure locus under interface-controlled failure}

Once the potential of computational micromechanics to predict the failure locus has been proved, this methodology can be used to assess the behavior under different loading conditions or fracture micromechanisms. A relevant example is the case of composites in which failure under transverse compression and shear is controlled by interface decohesion rather than matrix yielding. The mechanical behavior of the lamina subjected to different biaxial loading paths characterized by $55_{5}$ is plotted in Fig. 8 in the $a_{2}-t 12$ stress space for a composite lamina in which the interface strength was reduced to $t_{c}=0.5 \mathrm{c}=39.8 \mathrm{MPa}$ (to ensure that failure controlled by interface decohesion). The rest of the matrix, fiber and interface properties were not changed. As in the composites with a strong interface, simulations with different fiber realizations led to very little scatter in the maximum strength (below $6 \%$ between the maximum and minimum values). The composite strength in compression and shear was reduced (as compared to the composite with a strong interface, Fig. 5) and the reduction in the shear stress (indicative of shear

2 It should be noted that Puck failure criterion is equivalent to the more recent LaRCO4 failure criteria [20].

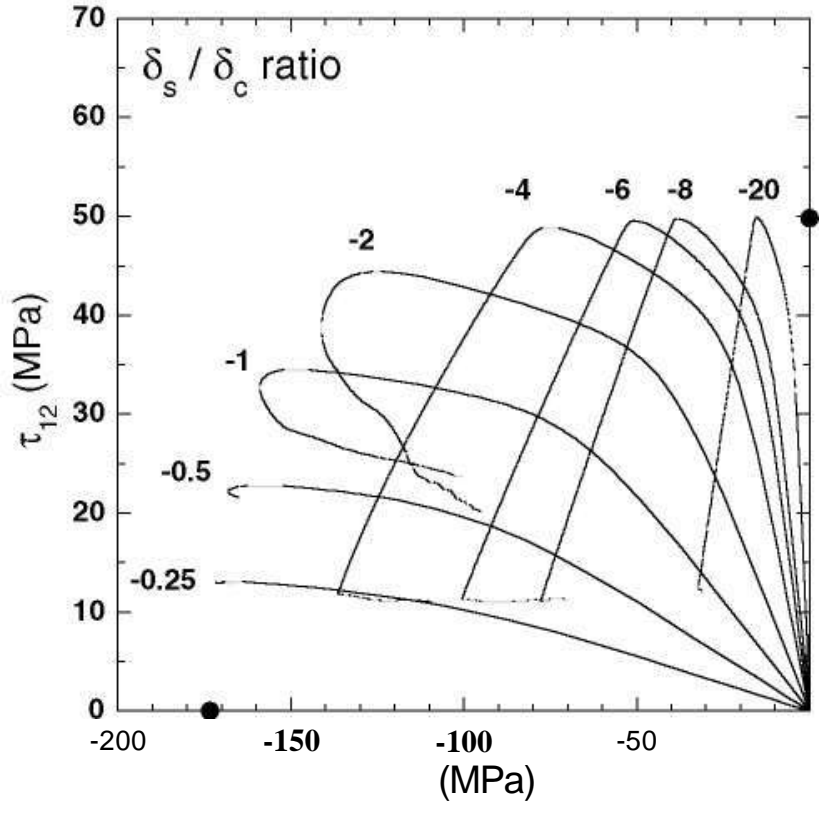

Fig. 8. Mechanical response of the composite lamina under biaxial deformation in the $<\mathrm{T}_{2}-\mathrm{T} 12$ stress space in the case of interface-controlled failure. The corresponding $15 \sqrt{15} 5_{\mathrm{s}}$ ratio for each curve is given in the figure. The solid circles correspond to the strength under uniaxial compression and longitudinal shear.

failure) after the peak load was abrupt in tests carried out under limited amount of compression.

The corresponding failure mechanisms in composite lamina with a weak interface subjected to uniaxial compression, biaxial compression and shear $\left(5_{\mathrm{C}} / 5_{\mathrm{S}}=-2\right)$ and longitudinal shear can be found in Fig. 9(a), (b) and (c), respectively, which shows the contour plots of the plastic strain at the peak load. Two main differences are found with respect to the composite with a strong interface. Shearfailure, which is dominant when $S J S_{S}<-3$, occurs by interface fracture in shear rather than matrix shear yielding, Fig. 9(c). As the interface shear strength is independent of the compressive normal stresses, the shear strength of the composite in this region does not depend on the compressive stresses and the composite strength is practically constant in the range $-3>S J S_{S}<\multimap$. The shape of the failure locus in this area is thus different from the one found in composites with a strong interface. Nevertheless, failure also occurs by the formation of a crack parallel to the applied shear stress $T_{12}$ formed by the link-up of interface cracks following the weakest path through the interfaces, Fig. 9(c). Under compression-dominated failure $\left(5_{\mathrm{C}} / 5_{\mathrm{S}}>-1\right.$, damage began by the nucleation of interface cracks at the equator of the fibers, perpendicularly to the compression axis, and continued by the formation of plastic shear bands in the polymeric matrix which linked-up the interface cracks. Thus, matrix damage was diffuse and it was not always possible to find a shear band which crossed the entire RVE, Fig. 9(a). An intermediate situation between both mechanisms was found at $5 \times 58=-2$, Fig. 9(b), in which features of compression- and sheardominated failure can be found.

Finally, the failure locus predicted by the numerical simulations for a composite with a weak interface is compared with the predictions of Puck and LaRC models in Fig. 10. The latter were obtained as before from the values of $\mathrm{S}_{\mathrm{L}}$ and $Y_{c}$ provided by the numerical simulations and assuming that $O_{O}=53^{\circ}$. As it was expected, both models - which are obtained under the hypothesis of matrix-dominated failure - are not able to predict failure surface. The largest differences are found in the shear-dominated region because they predict an increase in the composite strength as the applied com- 


\section{a}

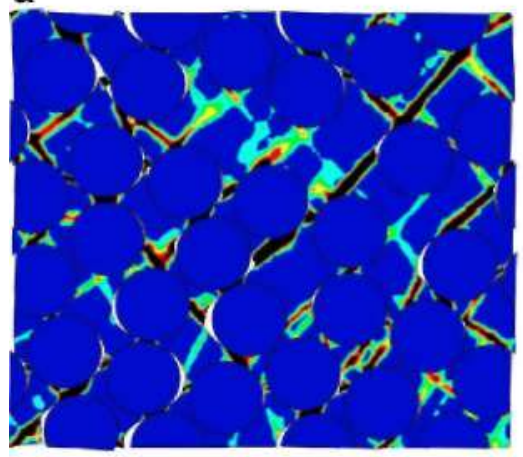

b

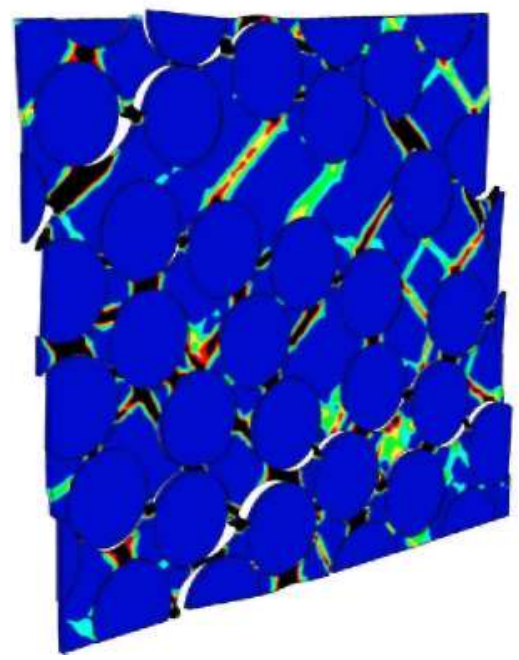

Plastic strain

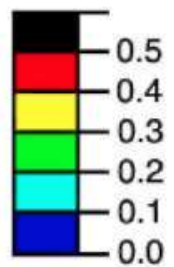

C

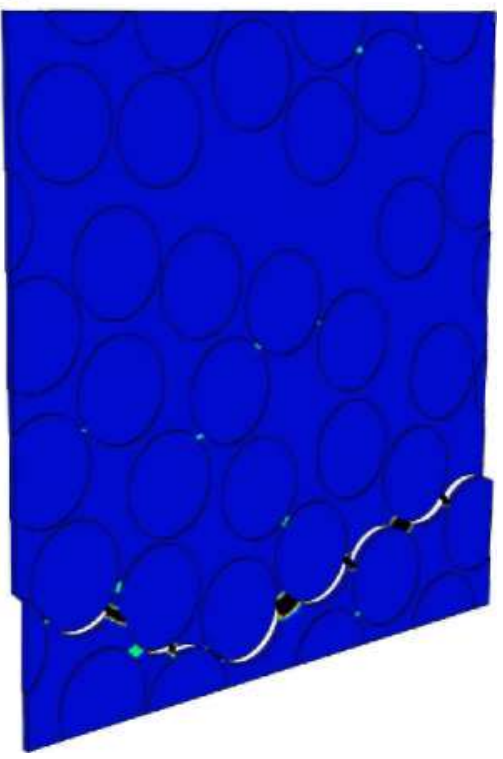

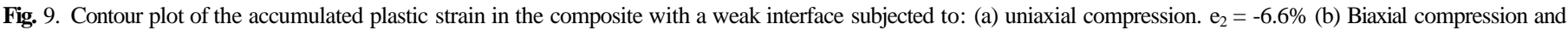

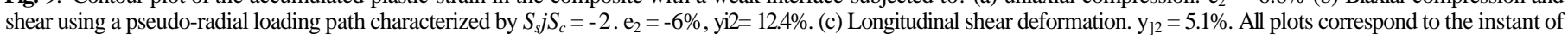
maximum stress in stress-strain curves of Fig. 8. Compression is always in the vertical direction.

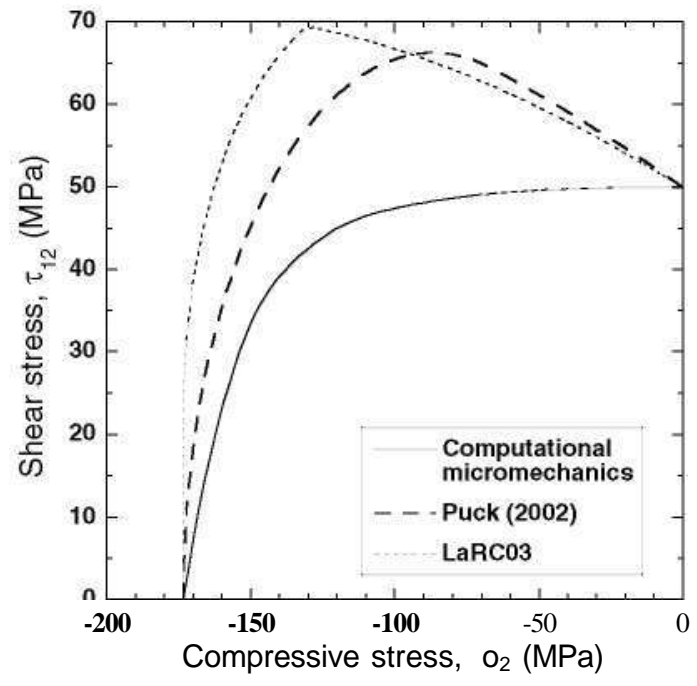

Fig. 10. Failure locus under transverse compression and longitudinal shear in the case of interface-controlled failure. Numerical simulations using computational micromechanics as well as predictions of Puck and LaRCO3 models.

pressive stress increases which is not realistic in the case of interface dominated failure. This result shows the potential of computational micromechanics simulations to provide guidance in the development of accurate failure criteria which take into account the physical processes of deformation and failure.

\section{Conclusions}

The mechanical response of a composite lamina made up of PEEK matrix unidirectionally reinforced with 60 vol\% C fibers subjected to transverse compression and longitudinal shear was simulated using computational micromechanics. The failure locus given by the envelope of the loading curves under different biaxial loading conditions in the $a_{2}-i \lambda i$ stress space - was in excellent agreement with the experimental results reported by Vogler and Kyriakides [1] for this material. In addition, the transition from shear to compression-dominated fracture and the differences in the orientation of the fracture plane were adequately predicted by the numerical simulations. These results demonstrate the actual potential of computational micromechanics to predict the macroscopic properties of composite lamina from the properties and spatial distribution of the constituents.

The numerical simulations were also useful to establish the range of validity of current failure criteria for composites. In particular, they showed that Puck failure criterion provides an excellent estimate for the failure locus of unidirectional laminates subjected to transverse compression and longitudinal shear when fracture is controlled by the matrix. This is not, however, the case in composites with a weak fiber/matrix interface in which interface 
decohesion, rather than matrix shear yielding, controls the mechanical behavior. The failure loci obtained from the computational micromechanics can be very useful in extending the accuracy of current failure criteria for conditions of interface dominated failure.

This investigation was supported by the Ministerio de Eduacion y Ciencia de Espana through the Grant MAT 2006-2602 and by the Comunidad de Madrid through the program ESTRUMAT-CM (reference MAT/0077).

\section{References}

Vogler TJ, Kyriakides S. Inelastic behavior of an AS4/PEEK composite under combined transverse compression and shear. Part I: Experiments. Int J Plasticity 1999;15:783-806

Zohdi TI, Wriggers P. Introduction to computational micromechanics. Berlin: Springer; 2005

Cailletaud G, Forest S, Jeulin D, Feyel F, Galliet I, Mounoury V, et al. Some elements of microstructural mechanics. Computat Mater Sci 2003:27:351-74. Gonzalez C, LLorca J. Multiscale modeling of fracture in fiber-reinforced composites. Acta Mater 2006;54:4171-81.

Gonzalez C, LLorca J. Numerical simulation of the fracture behavior of $\mathrm{Ti}$ / SiCcomposites between $20^{\circ} \mathrm{C}$ and $400{ }^{\circ} \mathrm{C}$. Metall Mater Trans A 2007;38: 169-79.

Delannay L, Jacques PJ, Kalindi SR. Finite element modeling of crystal plasticity with grains shaped as truncated octahedrons. Int J Plasticity 2006;22:1879-98. Youssef S, Maire E, Gaertner R. Finite element modelling of the actual structure of cellular materials determined by X-ray tomography. Acta Mater 2005:53:719-30.

Moraleda J, Segurado J, LLorca J. Finite deformation of porous elastomers: acomputational micromechanics approach. Philos Mag 2007;87:5607-27. Segurado J, Gonzalez C, LLorca J. A numerical investigation of the effect of particle clustering on the mechanical properties of composites. Acta Mater 2003;51:2355-69.

Segurado J, LLorca J. Computational micromechanics of composites: the effect of particle spatial distribution. Mech Mater 2006;38:873-83.

Chawla N, Sidhu RS, Ganesh W. Three-dimensional visualization and microstructure-based modeling of deformation in particle-reinforced composites. Acta Mater 2006;54:1541-8.

Miravete A, Bielsa JM, Chiminelli A Cuartero J, Serrano S, Tolosana N, et al. 3D mesomechanical analysis of three-axial braided composite materials. Compos Sci Technol 2006;66:2594-964.

Song S, Waas AM, Shahwan KW, Xiao X, Faruque O. Braided textile composites under compressive loads, modeling the response, strength and degradation. Compos Sci Technol 2007;67:3059-70.

Gonzalez C, LLorca J. Mechanical behavior of unidirectional fiber-reinforced polymers under transverse compression: microscopic mechanisms and modeling. Compos Sci Technol 2007;67:2795-806.
Hinton MJ, Soden PD, Kaddour AS. Failure Criteria in Fiber-reinforced Polymer Composites: The World Wide Failure Exercise. Amsterdam: Elsevier; 2004 Soden PD, Hinton MJ, Kaddour AS. A comparison of the predictive capabilities of current failure theories for composite laminates. Compos Sci Technol 1998;58:1225-54

Kaddour AS, Hinton MJ, Soden PD. A comparison of the predictive capabilities of current failure theories for composite laminates: additional contributions Compos Sci Technol 1998;58:1225-54.

Puck A, Schurmann H. Failure analysis of FRP laminates by means of physically based phenomenological models. Compos Sci Technol 1998;58:1045-67.

Puck A, Schurmann H. Failure analysis of FRP laminates by means of physically based phenomenological models. Compos Sci Technol 2002;62:1633-62.

Pinho ST, Davila CG, Camanho PP, Iannucci L, Robinson L. Failure models and criteria for FRP under in-plane of three-dimensional stress states including shear non-linearity. Report NASA/TM-2005-213530, 2005.

Hsu SY, Vogler TJ, Kyriakides S. Inelastic behavior of an AS4/PEEK composite under combined transverse compression and shear. Part II: Modeling. Int J Plasticity 1999;15:807-36.

Totry E, Gonzalez C, LLorca J. Influence of the loading path on the strength of fiber-reinforced composites subjected to transverse compression and shear. Int J Solids Struct 2008;45:1663-75

Segurado J, LLorca J. A numerical approximation to the elastic properties of sphere-reinforced composites. J Mech Phys Solids 2002;50:2107-21.

Totry E, Gonzalez C, LLorca J. Failure locus of fiber-reinforced composites under transverse compression and out-of-plane shear. Compos Sci Technol 2008;68:829-39.

Abaqus, Users' Manual, version 6.7, ABAQUS, Inc., 2008.

Gonzalez C, Segurado J, Lorca J. Numerical simulation of elasto-plastic deformation of composites: evolution of stress microfields and implications for homogenization models. J Mech Phys Solids 2004;52:1573-93.

Segurado J, LLorca J. A new three-dimensional interface finite element to simulate fracture in composites. Int J Solids Struct 2004;41:2977-93.

Abaqus, Theory Manual, HKS, Inc., 1998.

Menetrey $\mathrm{P}$, Willam KJ. Triaxial failure criterion for concrete and its generalization. AC StructJ 1995;92:311-8.

Quinson R Perez J, Rink M, Pavan A. Yield criteria for amorphous glassy polymers. J Mater Sci 1997;32:1371-9.

Hsu SY, Vogler TJ, Kyriakides S. Compressive strength predictions for fiber composites. J Appl Mech 1998;65:7-16.

Gregory JR, Spearing SM. Nanoindentation of neat and in situ polymers in polymer-matrix composites. Compos Sci Technol 2005;65:595-607.

Segurado J, LLorca J. A computational micromechanics study of the effect of interface decohesion on the mechanical behavior of composites. Acta Mater 2005:53:4931-42.

Zhou X-F, Wagner HD, Nutt SR. Interfacial properties of polymer composites measured by push-out and fragmentation tests. Composites A 2001:32:1543-51.

Davila C, Camanho PP, Rose CA. Failure criteria for FRP laminates. J Compos Mater 2005;39:323-45.

Pinho ST, Iannucci L, Robinson P. Physically-based failure models and criteria for laminated fibre-reinforced composites with emphasis on fibre-kinking. Part I: Development. Composites A 2006;37:63-73. 